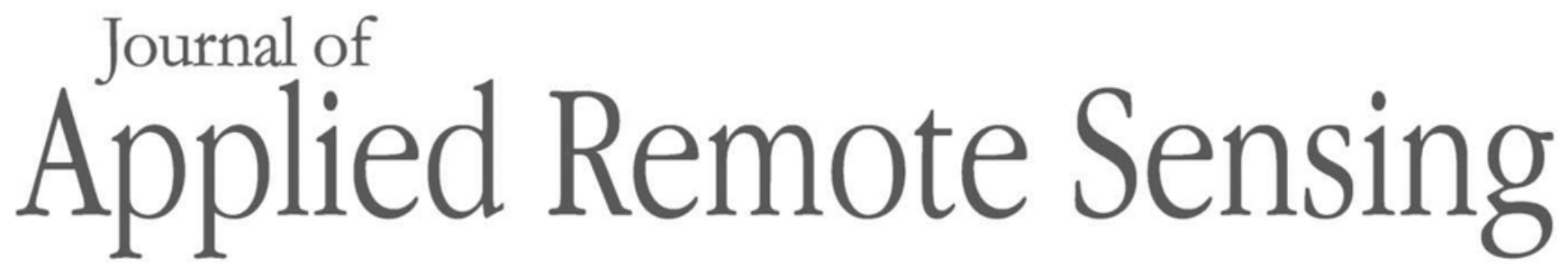

RemoteSensing.SPIEDigitalLibrary.org

\title{
Implementation of a multiangle soil moisture retrieval model using RADARSAT-2 imagery over arid Juyanze, northwest China
}

\author{
Liping Yang \\ Yanfei Li \\ Qi Li \\ Xiaohui Sun \\ Jinling Kong \\ Le Wang
}




\title{
Implementation of a multiangle soil moisture retrieval model using RADARSAT-2 imagery over arid Juyanze, northwest China
}

\author{
Liping Yang,* Yanfei Li, Qi Li, Xiaohui Sun, Jinling Kong, and Le Wang \\ Chang'an University, Department of Geographic Information Science, Xi'an, China
}

\begin{abstract}
Accurate retrieval of soil moisture is important for understanding regional environmental changes and sustainable development in arid regions. Through numerical simulation and regression analysis based on advanced integral equation model (AIEM), the study aims to establish a multiangle soil moisture retrieval model based on RADARSAT-2 image in arid Juyanze. A combined roughness parameter $R_{s}$ was established, and then the influences of roughness and soil moisture on the backscattering simulations were discussed. Finally, the empirical multiangle soil moisture retrieval model was implemented and validated in Juyanze. Inversion results show that the model has favorable validity. The coefficient of determination $R^{2}$ between the inferred and measured soil moisture is 0.775 with a root-mean-square error (rmse) of $0.626 \%$, implying better retrieval accuracy. Soil moisture varies from about $0.1 \%$ to $25 \%$ and is no more than $10 \%$ in most parts of this region, which is in reasonable agreement with the factual circumstances. The model directly relates the Fresnel reflection coefficient and soil moisture and is independent of ground roughness measurements. With a wider angular range, it has great potential for soil moisture evaluation in arid regions. (C) The Authors. Published by SPIE under a Creative Commons Attribution 3.0 Unported License. Distribution or reproduction of this work in whole or in part requires full attribution of the original publication, including its DOI. [DOI: 10.1117/1.JRS.11.036029]
\end{abstract}

Keywords: soil moisture; retrievals; RADARSAT; advanced integral equation model; Juyanze; arid China.

Paper 170240 received Mar. 24, 2017; accepted for publication Aug. 29, 2017; published online Sep. 23, 2017.

\section{Introduction}

Soil moisture is a crucial state variable within the fields of hydrology, climatology, ecology, and agriculture. ${ }^{1-4}$ In arid and semiarid regions, soil moisture is the most sensitive environmental factor. Accurate estimation of soil moisture in arid and semiarid regions not only improves our understanding on the regional hydroclimatic processes but also provides basic data for solutions to many environmental and ecological problems.

Although traditional ground-based measurements are the most accurate methods for soil moisture estimation, such techniques are often rather complex, expensive, labor-intensive, and limited to discrete measurements. The advent of satellite-based remote sensing has greatly facilitated the acquisition of various land surface parameters at a variety of scales in wide ranges and with high accuracy without expensive in-situ monitoring networks. ${ }^{5}$ Advances in remote sensing technology have shown that soil moisture at the surface layer (i.e., 0 to $5 \mathrm{~cm}$ of the land surface) can be measured to some degree by all regions of the electromagnetic radiation spectrum. ${ }^{6}$ Microwave remote sensors can operate day and night in nearly all weather conditions and thus have been extensively utilized to retrieve soil moisture across a broad range of scales. As the most common imaging active microwave configuration, synthetic aperture radar (SAR) uses a self-contained source of microwave radiation to illuminate the surface and measure the amount of radiation returned to the sensors. ${ }^{7}$ This allows SAR to monitor soil moisture with relatively high prediction accuracy.

*Address all correspondence to: Liping Yang, E-mail: zylpyang@chd.edu.cn 
Various models, ranging from physically based models to experimental relationships, have been developed to invert the backscattering coefficient to soil moisture over the past decades. ${ }^{8}$ The most popular physically based models are the small perturbation model, ${ }^{9}$ Kirchhoff approximations,${ }^{10}$ the small slope approximation (SSA) model, ${ }^{11}$ and the integral equation model (IEM), ${ }^{12,13}$ as well as its amended version, the advanced integral equation model (AIEM). ${ }^{14}$ Theoretical models can be used in a wide variety of conditions while making few priori assumptions about the characteristics of the surface. ${ }^{5}$ The simulation to backscatter is also relatively accurate. For bare surface soil, the IEM/AIEM has become the most widely used scattering model..$^{15}$ Nevertheless, theoretical models, being more mathematically complicated, were usually used as a base to develop empirical or semiempirical models. Although many empirical or semiempirical models have been developed ${ }^{16-19}$ it is generally not possible to apply relationships observed over one area for radar backscatter inversion in other areas.

As radar return from bare soil is mainly governed by the soil dielectric properties (which are strongly dependent on the free water content in the soil) and surface roughness, ${ }^{1}$ an exact and complete description of the surface roughness of a soil medium is essential to the accurate analysis and interpretation of backscattering behavior and soil moisture estimation. Although diverse approaches for the improvement of roughness descriptions have been proposed, ${ }^{20-23}$ the availability of a limited number of radar configurations makes it generally impossible to retrieve the soil moisture with all of the roughness parameters. In this context, a description written in the form $Z_{s}=S^{2} / l$ was introduced ${ }^{24}$ to mix the effects of the root mean square (rms) height $S$ and the correlation length $l$ on the values of $\sigma$. Various revisions and improvements were also reported. ${ }^{25-27}$ Before the combined roughness description can be widely applied, however, additional research over arid regions must be conducted.

In this paper, through numerical simulation and regression analysis based on AIEM, a combined roughness parameter $R_{s}$ was established to eliminate the effect of surface roughness on the soil moisture inversion. Influences of roughness and soil moisture on the backscattering simulations were discussed. A multiangle soil moisture retrieval model, which directly relates the Fresnel reflection coefficient and soil moisture and is independent of ground roughness measurements, was implemented and validated in Juyanze for the first time. The paper is organized into six sections. The study area and data are briefly introduced in Sec. 2. Section 3 describes the methods. Section 4 presents the results and validation. Discussions are given in Sec. 5. Finally, conclusions are presented in Sec. 6.

\section{Study Area and Data}

\subsection{Study Area}

Juyanze is located at the lower reach of the Heihe River in Inner Mongolia, China. A southnorth-striking structure separates Juyanze into the east and west parts (Fig. 1). Juyanze used to be connected to Gaxun Nur and Sogo Nur and formed mega-paleolakes during historical periods. ${ }^{28}$ Currently, the Juyanze Basin only contains small shallow water bodies fed by groundwater and sporadic river discharge. Intensive land use and irrigation in the upper reaches of the Heihe River (the densely populated "Hexi corridor") has reduced modern flow to these terminal lakes. ${ }^{29}$ The annual mean temperature is $8.3^{\circ} \mathrm{C}$. The annual mean precipitation and evaporation are 37 and $3841.81 \mathrm{~mm}$, respectively. Under such an extremely dry climate, the vegetation cover is very low and sandy lands, Gobi, bare soil, saline-alkali soil, bare rocks, low-coverage grasslands, and shallow lakes form the regional landscapes.

\subsection{Satellite Data and Preprocessing}

During field investigations (July 4-12, 2014), a fully polarimetric RADARSAT-2 SAR image was acquired on July 6, 2014, at C-band frequency $(5.405 \mathrm{GHz})$ with an incidence angle of $20.17 \mathrm{deg}$ and a spatial resolution of $8 \mathrm{~m}$. The nominal coverage was $25 \times 25 \mathrm{~km}$. A Landsat 8 operational land imager (OLI) image acquired on July 8, 2014, and a SRTM DEM data were also used as ancillary data. 


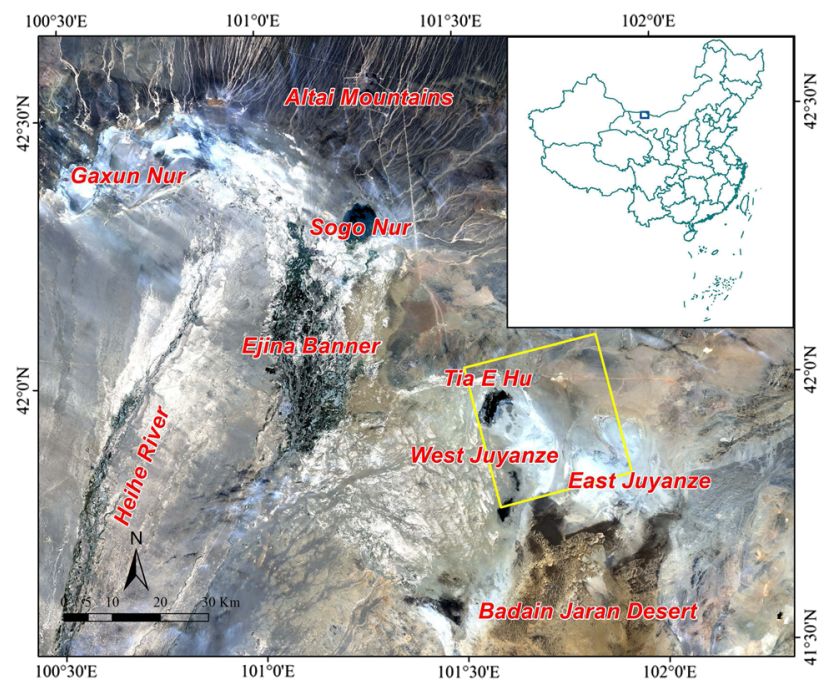

Fig. 1 The location of Juyanze. The blue rectangle in the insert map shows the location of Juyanze in inner Mongolia, China. The yellow rectangle indicates the area of the RADARSAT-2 image acquired on July 6, 2014.

The SAR image was first calibrated to convert pixels from single-look complex data to a multilook image using the NEST. A $5 \times 5$ refined Lee filtering was then applied. By radiometric calibration, the backscattering coefficient $(\sigma)$ in decibels $(\mathrm{dB})$ was transformed from the DN of each pixel. Thereafter, range Doppler terrain correction was performed based on the SRTM $3 \mathrm{~s}$. The average backscattering coefficients were calculated using NEST.

\subsection{Ground Measurements and Analysis}

Coincident with SAR overpass, three sampling lines and 50 sampling sites were selected for the representativeness of land cover and topography, as well as the feasibility for field sampling. The sampling sites are shown on the Landsat 8 false color composite image (Fig. 2).

The top $5 \mathrm{~cm}$ of the surface soil was sampled using aluminum boxes for moisture and texture characterization analysis. Soil moisture was measured using the Gravimetric method. In the study area, the volumetric soil moisture $\left(M_{v}\right)$ ranges from $0.31 \%$ to $10.33 \%$, and the average

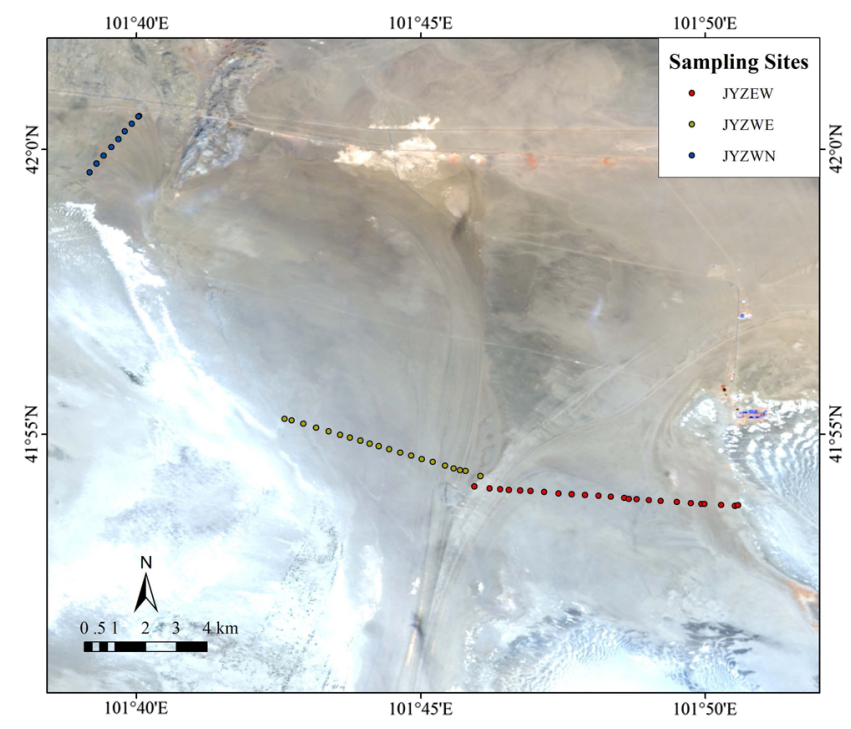

Fig. 2 Landsat 8 OLI color composite image (band $4=$ red, band $3=$ green, and band $2=$ blue) acquired on July 8, 2014. Red, green, and blue dots indicate the sampling sites at the west part of east Juyanze and the east and north parts of west Juyanze, respectively. 
value is $2.24 \%$. Soil texture characterization was analyzed using a mastersizer 2000 laser particle size analyzer. The percentages of sand, silt, and clay are $83.87 \%, 13.77 \%$, and $2.37 \%$, respectively. The soil is sandy soil and is composed of loamy sand.

Surface roughness was measured using a needle profilometer (2-m long and with 1-cm sampling intervals) and a digital camera. At each sampling site, four field photographs were taken; two were along the row direction, and the rest were across. The photographs were processed by a GetData Graph Digitizer. The rms height $S$ and correlation length $l$ range from 0.2 to $2.4 \mathrm{~cm}$ and 3 to $33 \mathrm{~cm}$, respectively, in the study area.

\section{Methods}

Based on the simulation of backscattering characteristics using AIEM, influences of roughness and soil moisture on the backscattering simulations were discussed. Finally, a multiangle empirical soil moisture retrieval model was implemented and validated.

\subsection{Advanced Integral Equation Model Simulation}

Since most natural terrain has a small rms slope, single scattering will dominate over the multiple scattering in most situations. ${ }^{2}$ The single backscattering coefficients from AIEM have been well described, ${ }^{14,30,31}$ and the equations are not reprinted here.

The AIEM input parameters were determined according to RADARSAT-2 specifications and the measured data. The soil bulk density is $1.6147 \mathrm{gcm}^{-3}$. The percentage of sand and clay is $83.87 \%$ and $2.37 \%$, respectively, and the temperature is about $30^{\circ} \mathrm{C}$ when sampling. A powerlaw spectrum correlation function was chosen to simulate the scatter characteristics. As shown in Table 1, a backscattering simulated database, which was used to implement the empirical model, was set up.

\subsection{Analysis of Simulated Backscattering as Functions of Roughness and Soil Moisture}

The backscattering coefficients of a bare soil surface can be presented as a product of two functions: $2,3,32,33$

$$
\sigma_{\mathrm{qp}}=A\left(R_{s}, \theta\right) \cdot R(\Gamma, \theta) .
$$

One is the roughness function $A$ that describes the effect of the surface roughness. The other is the dielectric function (soil moisture function) $R$ that is commonly related to the Fresnel reflection coefficients $\Gamma$. $\sigma_{\mathrm{qp}}$ represents the backscattering coefficient and the subscripts $q$ and $p$ denote the transmitting and receiving polarization, respectively. Meanwhile, the two functions are independent of each other. Their relationships with the backscattering coefficients will be analyzed independently, and the mutual effects will be further discussed.

\subsubsection{Influence of roughness on the backscattering simulations}

The rms height $S$ and the correlation length $l$ are two popular parameters used to depict the surface roughness. Previous research demonstrated that higher accuracy in the soil moisture

Table 1 Parameters setting in AIEM.

\begin{tabular}{lcccc}
\hline \hline Parameters & Minimum & Maximum & Interval & Unit \\
\hline Incidence angle $\theta$ & 15 & 50 & 2 & Degree \\
Soil moisture $M_{v}$ & 1 & 30 & 2 & \% by volume \\
Rms height $S$ & 0.2 & 2.4 & 0.2 & Centimeter \\
Correlation length I & 3 & 33 & 3 & Centimeter \\
\hline \hline
\end{tabular}


Yang et al.: Implementation of a multiangle soil moisture retrieval model using RADARSAT-2 imagery...

Table 2 Relationship between the backscattering coefficients and the roughness parameters.

The regression relationship between

backscattering coefficient $\sigma$ and

roughness parameters $S$ and $I$

Coefficient of determination

Modified

$\sigma_{\mathrm{Vv}}=9.636 \ln (S)-6.495 \ln (I)+5.383$

$R^{2}=0.982$

$\sigma_{\mathrm{VV}} \approx 3.23 \ln \left(\frac{S^{3}}{R^{2}}\right)+5.383$

$\sigma_{\mathrm{HH}}=10.930 \ln (S)-6.457 \ln (I)+4.953$

$R^{2}=0.986$

$\sigma_{\mathrm{HV}}=12.802 \ln (S)-6.495 \ln (I)+6.543$

$R^{2}=0.989$

$\sigma_{\mathrm{HH}} \approx 3.44 \ln \left(\frac{S^{3}}{l^{2}}\right)+4.953$

$\sigma_{\mathrm{VH}}=9.636 \ln (S)-7.008 \ln (I)+6.315$

$R^{2}=0.991$

$\sigma_{\mathrm{HV}} \approx 3.76 \ln \left(\frac{S^{3}}{I^{2}}\right)-6.543$

$\sigma_{\mathrm{VH}} \approx 3.81 \ln \left(\frac{S^{3}}{l^{2}}\right)-6.315$

estimation could be obtained using the combined roughness parameters, and several roughness parameters combining the two parameters have been put forward. ${ }^{2,24-27}$ These results are particularly useful for the improvement of empirical or semiempirical inversion models used in soil moisture estimations because in the past these models were often based on the rms height $S$ only, leading to a high level of noise and a lower accuracy in the soil moisture estimation. ${ }^{24,26}$ To mix the effect of rms height $S$ and the correlation length $l$, a combined roughness parameter $R_{s}$ was established in this study.

According to numerical simulations on the relationships between the backscattering coefficients and the rms height $S$ as well as the correlation length $l$, similar functional relationships were detected in four polarizations in our study (Table 2). The ratio between the coefficients of $S$ and $l$ in column 1 of Table 2 is about 3 to 2 . By reorganizing the original functions, modified functions including $R_{s}=S^{3} / l^{2}$, defined as the combined roughness parameter $R_{s}$, were taken in our study.

Figure 3 shows scatter plots of the backscattering coefficient as a function of $R_{s}$ in four polarizations. The blue points are from the AIEM simulation, while the black lines are the fitted curve by logarithm functions. It shows a sharp increase of backscattering with $R_{s}$ logarithmically and then saturation. All coefficients of determination $R^{2}$ could reach 0.936 , indicating a strong correlation between the backscattering coefficients and $R_{s}$. Based on this, an approximation form for $\sigma_{\mathrm{qp}}$ as a function of $R_{s}$ was found and can be written as

$$
\sigma_{\mathrm{qp}}=A \ln \left(R_{S}\right)+B
$$

where $A$ and $B$ are estimated through regression analysis.

\subsubsection{Influence of soil moisture on the backscattering simulations}

Relationship between the backscattering coefficient and soil moisture is rather complex, while relationship between the Fresnel reflection coefficient and backscattering coefficient is more direct. Further analysis between the Fresnel reflection coefficient and dielectric constant could be used to calculate soil moisture content. Hence, the relationship between the Fresnel reflection coefficient and backscattering coefficient is analyzed based on AIEM simulated database under the following conditions: the incidence angle $\theta$ is $20.17 \mathrm{deg}$, the frequency $f$ is $5.405 \mathrm{GHz}$, the rms height $S$ is $0.6139 \mathrm{~cm}$, and the correlation length $l$ is $16.8735 \mathrm{~cm}$, $M_{v} \in(0.01,0.30)$ at intervals of $1 \%$.

The simulation results in four polarizations are demonstrated in Fig. 4, where the blue points are from the AIEM simulation and the black lines are the fitted curve by logarithm functions. The results show that, with the increase of the Fresnel reflection coefficient $\Gamma$, the backscattering coefficient increases logarithmically and a very strong correlation is observed between them with $R^{2}$ larger than 0.999 . Figure 5 is the fitted curve between $\Gamma$ and $M_{v}$, and the $R^{2}$ is 0.999 , indicating a very strong correlation between them.

Thus, approximation forms for $\sigma_{\mathrm{qp}}$ as a function of $\Gamma$ and $M_{v}$ as a function of $\Gamma$ could be expressed as

$$
\sigma_{\mathrm{qp}}=C \ln (\Gamma)+D
$$




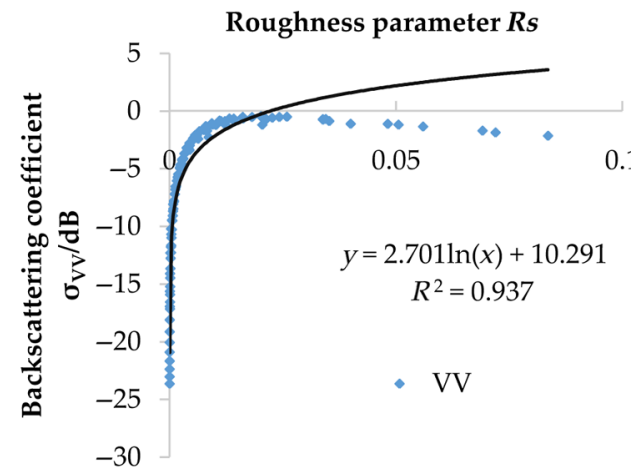

(a)

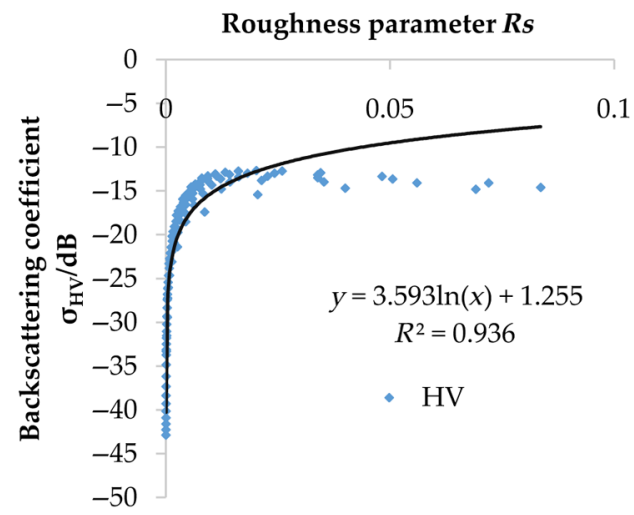

(c)

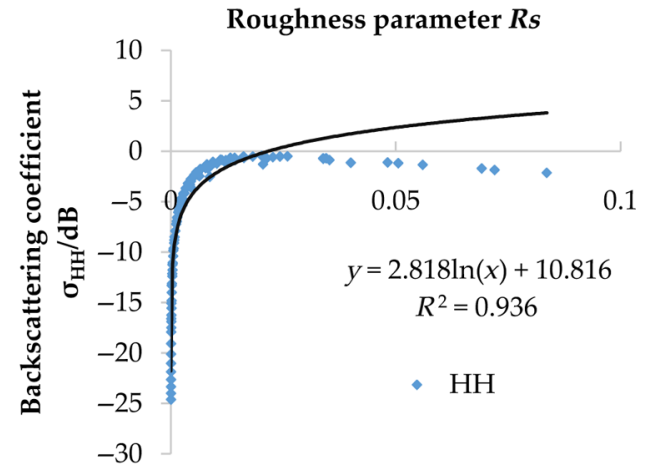

(b)

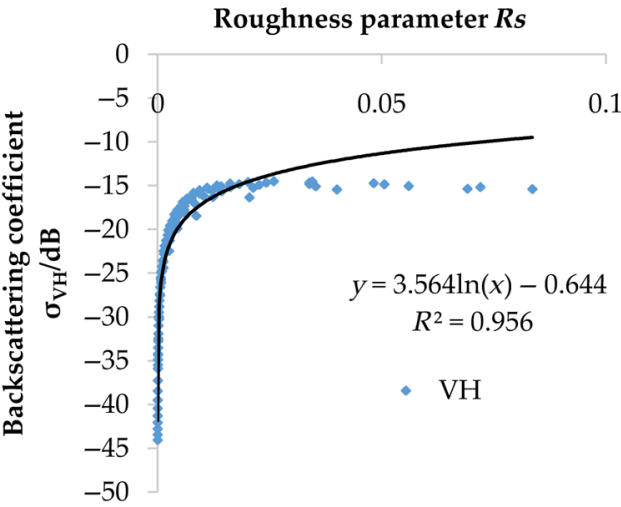

(d)

Fig. 3 Relationship between the backscattering coefficients and combined roughness parameter in four polarizations, where the blue points are from the AIEM simulation, while the black lines are the fitted curve by logarithm functions: (a) VV $\left(R^{2}=0.937\right)$, (b) $\mathrm{HH}\left(R^{2}=0.936\right)$, (c) $\mathrm{HV}$ $\left(R^{2}=0.936\right)$, and (d) $\mathrm{VH}\left(R^{2}=0.956\right)$.

$$
M_{V}=E \Gamma^{2}+F \Gamma+G
$$

where $C, D, E, F$, and $G$ can be estimated through regression analysis, $M_{v}$ represents soil moisture, and $\Gamma$ is the Fresnel reflection coefficient on the normal direction, which can be calculated by the following equation: ${ }^{18}$

$$
\Gamma=\left|\frac{1-\sqrt{\varepsilon}}{1+\sqrt{\varepsilon}}\right|^{2},
$$

where $\varepsilon$ is the dielectric constant and can be estimated through the Dobson model. ${ }^{16}$

\subsection{Implementation of the Empirical Model}

Based on above analyses, taking the roughness parameter and the dielectric constant (soil moisture) into account simultaneously, at a specific incidence angle, Eqs. (2) and (3) can be combined as follows (take VV as an example):

$$
\sigma_{\mathrm{vv}}=\{[\mathrm{A} \ln (R \mathrm{~s})+\mathrm{B}] \times[\mathrm{C} \ln (\Gamma)+\mathrm{D}]\} .
$$

In other polarizations, similar relationships between the backscattering coefficient and both $R_{s}$ and $\Gamma$ are detected; the equations are not shown here.

In the following steps, Eq. (6) was further spread and combined with Eq. (4). An empirical model at a specific incidence angle expressed as Eqs. (7) and (8) was established to describe the backscattering characteristics of bare surface as a function of $R_{s}$ and $\Gamma$. 
Yang et al.: Implementation of a multiangle soil moisture retrieval model using RADARSAT-2 imagery...

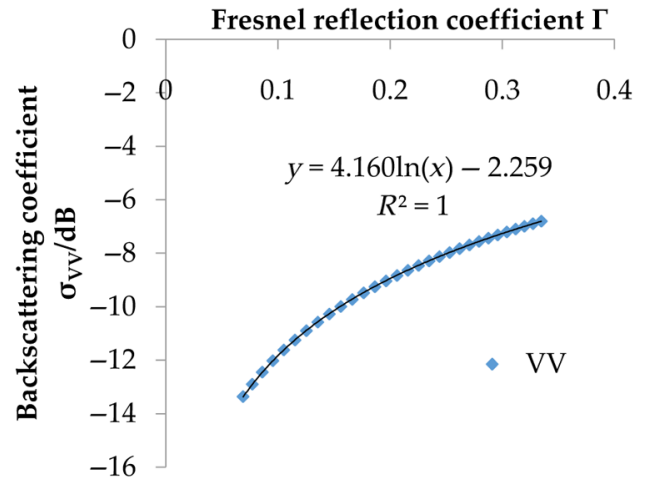

(a)

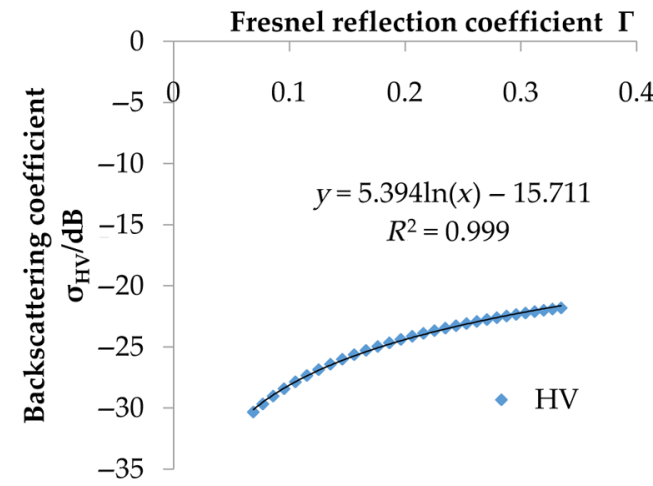

(c)

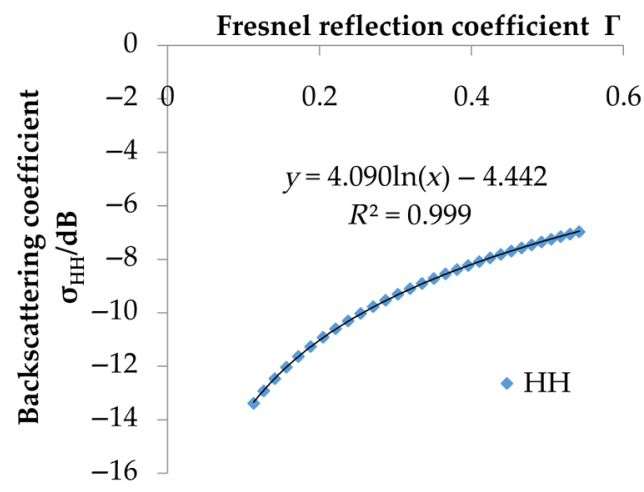

(b)

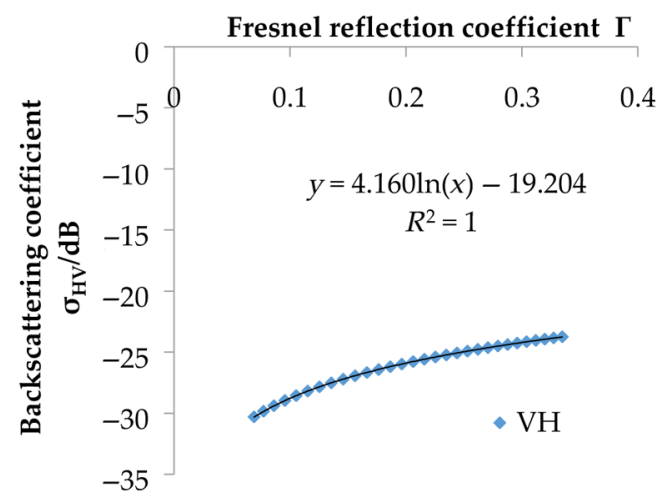

(d)

Fig. 4 Relationship between the backscattering coefficients and Fresnel reflection coefficient in four polarizations, where the blue points are from the AIEM simulation, while the black lines are the fitted curve by logarithm functions: (a) $\mathrm{VV}\left(R^{2}=1\right)$, (b) $\mathrm{HH}\left(R^{2}=0.999\right)$, (c) $\mathrm{HV}\left(R^{2}=0.999\right)$, and (d) $\mathrm{VH}\left(R^{2}=1\right)$.

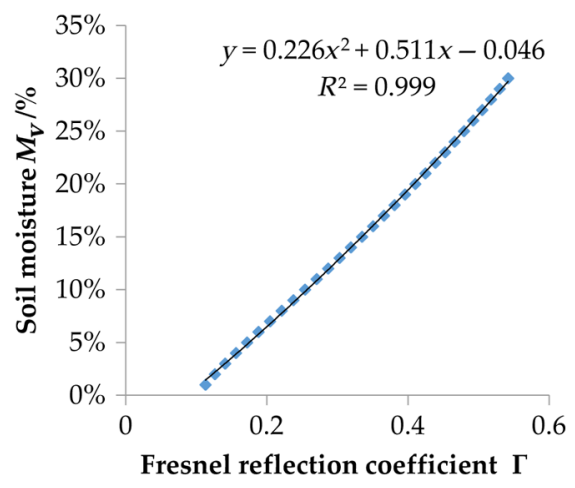

Fig. 5 Relationship between the Fresnel reflection coefficient and soil moisture $\left(R^{2}=0.999\right)$, where the blue points are from the AIEM simulation, while the black lines are the fitted curve.

$$
\begin{gathered}
\sigma_{\mathrm{qp}}=\alpha \ln \left(R_{s}\right)+\beta \ln (\Gamma)+\gamma \ln \left(R_{s}\right) \ln (\Gamma)+\delta, \\
M_{V}=\rho \Gamma^{2}+\tau \Gamma+\varphi .
\end{gathered}
$$

In the above equations, $\alpha, \beta, \gamma, \delta, \rho, \tau$, and $\varphi$ are coefficients determined by the least-square regression. When two polarization measurements are available, the influence of $R_{s}$ can be eliminated. 


\section{Results and Validation}

\subsection{Reliability Test of the Empirical Model}

Forty-eight samples were randomly selected from the simulated database to calculate coefficients and empirical formulas. Take VV and $\mathrm{VH}$ polarizations as examples; at the incidence angle of $20.17 \mathrm{deg}$, the expressions are as follows:

$$
\begin{gathered}
\sigma_{\mathrm{VV}}=2.675 \ln \left(R_{s}\right)+4.167 \ln (\Gamma)+0.022 \ln \left(R_{s}\right) \ln (\Gamma)+15.063, \\
\sigma_{\mathrm{VH}}=4.345 \ln \left(R_{s}\right)+4.203 \ln (\Gamma)+0.043 \ln \left(R_{s}\right) \ln (\Gamma)+8.329 .
\end{gathered}
$$

Figure 6 shows a comparison of the backscattering coefficient generated from the empirical model versus those derived from AIEM. In VV and VH polarizations, the coefficients of determination $R^{2}$ are 0.972 and 0.982 , respectively, and have passed the significance test of 0.05 . The standard errors are 0.608 and 0.741 , respectively.

\subsection{Implementation of Multiangle Empirical Model}

The reliability test above clearly suggests that simulated values from the empirical model and AIEM compare fairly well at a specific incidence angle. Thus, taking VV and VH as examples, the empirical model was further extended in the study area at incidence angles ranging between $15 \mathrm{deg}$ and $50 \mathrm{deg}$ with the interval of $5 \mathrm{deg}$ to derive empirical coefficients at different incidence angles. Tables 3 and 4 are for $\mathrm{VV}$ and $\mathrm{VH}$ polarizations, respectively, where $R^{2}$ is the coefficient of determination.

Through the fitting of coefficients at different incidence angles, empirical coefficients $\alpha(\theta)$, $\beta(\theta), \gamma(\theta), \delta(\theta), \rho(\theta), \tau(\theta)$, and $\varphi(\theta)$ based on the incidence angle can be derived.

Finally, the multiangle empirical model in this study is expressed as

$$
\sigma_{\mathrm{qp}}=\alpha(\theta) \ln \left(R_{s}\right)+\beta(\theta) \ln (\Gamma)+\gamma(\theta) \ln (R \mathrm{~s}) \ln (\Gamma)+\delta(\theta),
$$

and

$$
M_{V}=\rho(\theta) \Gamma^{2}+\tau(\theta) \Gamma+\varphi(\theta)
$$

where $\alpha(\theta), \beta(\theta), \gamma(\theta), \delta(\theta), \rho(\theta), \tau(\theta)$, and $\varphi(\theta)$ are the coefficients, $R_{s}$ is the roughness parameter, $\Gamma$ is the Fresnel reflection coefficient, and $M_{v}$ is the soil moisture.

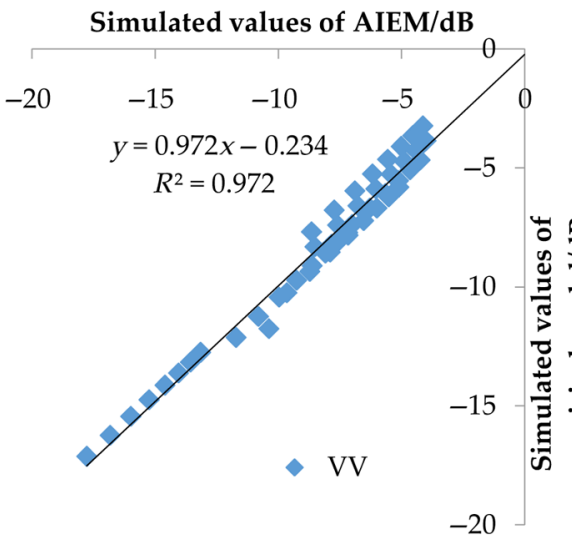

(a)

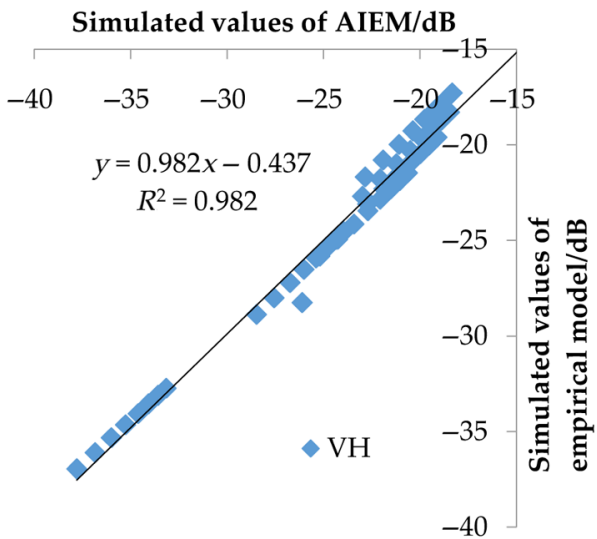

(b)

Fig. 6 Comparison of the simulated values between the empirical model and the AIEM: (a) VV $\left(R^{2}=0.972\right)$ and (b) $\mathrm{VH}\left(R^{2}=0.982\right)$. 
Yang et al.: Implementation of a multiangle soil moisture retrieval model using RADARSAT-2 imagery...

Table 3 Empirical coefficients at different radar incidence angles in VV polarization.

\begin{tabular}{ccccccccc}
\hline \hline$\theta$ & $\alpha(\theta)$ & $\beta(\theta)$ & $\gamma(\theta)$ & $\delta(\theta)$ & $\rho(\theta)$ & $\tau(\theta)$ & $\varphi(\theta)$ & $R^{2}$ \\
\hline 15 & 1.558 & 4.200 & -0.005 & 9.643 & 1.374 & 0.119 & -0.014 & 0.567 \\
20 & 2.067 & 4.086 & -0.009 & 9.697 & 1.378 & 0.152 & -0.015 & 0.746 \\
25 & 2.426 & 3.935 & -0.013 & 9.354 & 1.382 & 0.198 & -0.016 & 0.846 \\
30 & 2.689 & 3.745 & -0.018 & 8.791 & 1.383 & 0.259 & -0.017 & 0.906 \\
35 & 2.886 & 3.510 & -0.022 & 8.087 & 1.377 & 0.339 & -0.017 & 0.945 \\
40 & 3.038 & 3.224 & -0.025 & 7.275 & 1.352 & 0.445 & -0.016 & 0.966 \\
45 & 3.159 & 2.880 & -0.027 & 6.353 & 1.276 & 0.592 & -0.014 & 0.980 \\
50 & 3.262 & 2.461 & -0.027 & 5.278 & 1.044 & 0.807 & -0.009 & 0.986 \\
\hline \hline
\end{tabular}

Table 4 Empirical coefficients at different radar incidence angles in $\mathrm{VH}$ polarization.

\begin{tabular}{lcccccccc}
\hline \hline$\theta$ & $\alpha(\theta)$ & $\beta(\theta)$ & $\gamma(\theta)$ & $\delta(\theta)$ & $\rho(\theta)$ & $\tau(\theta)$ & $\varphi(\theta)$ & $R^{2}$ \\
\hline 15 & 2.490 & 4.200 & -0.005 & -2.016 & 1.374 & 0.119 & -0.014 & 0.753 \\
20 & 2.939 & 4.086 & -0.009 & -1.133 & 1.378 & 0.152 & -0.015 & 0.844 \\
25 & 3.259 & 3.935 & -0.013 & -0.791 & 1.382 & 0.198 & -0.016 & 0.900 \\
30 & 3.495 & 3.745 & -0.018 & -0.789 & 1.383 & 0.259 & -0.017 & 0.936 \\
35 & 3.674 & 3.510 & -0.022 & -1.024 & 1.377 & 0.339 & -0.017 & 0.961 \\
40 & 3.811 & 3.224 & -0.025 & -1.451 & 1.352 & 0.445 & -0.016 & 0.976 \\
45 & 3.922 & 2.879 & -0.027 & -2.061 & 1.276 & 0.592 & -0.014 & 0.986 \\
50 & 4.017 & 2.461 & -0.027 & -2.891 & 1.044 & 0.807 & -0.009 & 0.992 \\
\hline \hline
\end{tabular}

\subsection{Validation and Application of the Model}

Based on above-mentioned model, the RADARSAT- 2 image at an incidence angle of $20.17 \mathrm{deg}$ was used to validate the model and to retrieve soil moisture in Juyanze. Thirty samples were randomly selected as training samples, and the remaining 20 samples were used to validate the model. Substituting the values of 30 training samples into Eqs. (11) and (12), the empirical model in VV and $\mathrm{VH}$ can be written as

$$
\begin{aligned}
& \sigma_{\mathrm{VV}}=1.788 \ln \left(R_{s}\right)+16.400 \ln (\Gamma)+2.090 \ln \left(R_{s}\right) \ln (\Gamma), \\
& \sigma_{\mathrm{VH}}=4.592 \ln \left(R_{s}\right)+32.042 \ln (\Gamma)+4.684 \ln \left(R_{s}\right) \ln (\Gamma),
\end{aligned}
$$

and

$$
M_{v}=0.488 \Gamma^{2}-0.205 \Gamma+0.022 .
$$

Figure 7 shows a comparison of the inferred and measured soil moisture. The absolute errors are estimated to range from $0.024 \%$ to $1.747 \%$ in the 20 checkpoints. The $R^{2}$ is 0.775 with a root-mean-square error (rmse) of $0.626 \%$, implying a good correlation between the inferred and measured soil moisture. The model was applied to the RADARSAT- 2 image of this region, and the inferred soil moisture is displayed in Fig. 8. 


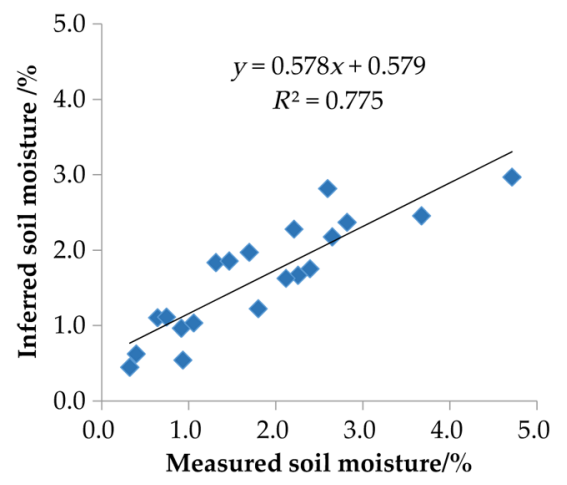

Fig. 7 Comparison between the inferred and ground-measured soil moisture in July 2014 $\left(R^{2}=0.775\right)$.

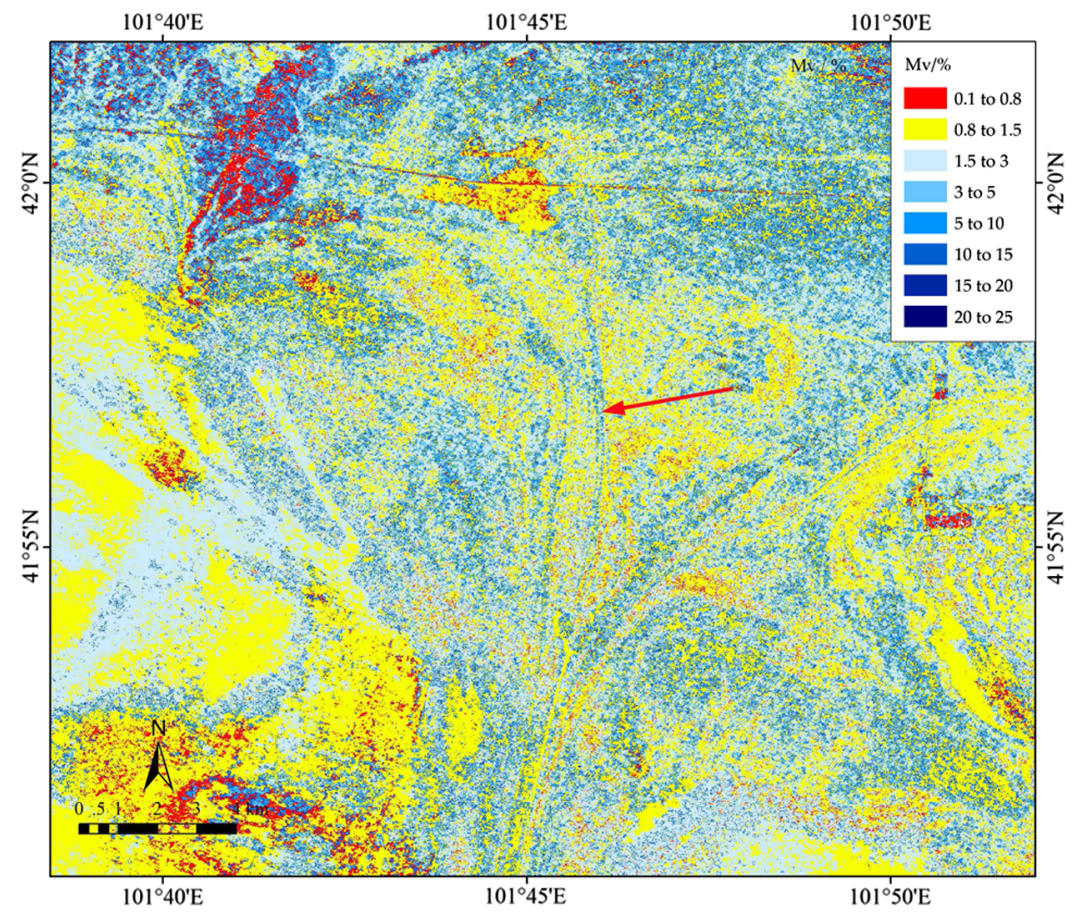

Fig. 8 The inferred volumetric soil moisture in \% derived from RADARSAT-2 image acquired on July 6,2014 . The area is about $22 \times 20 \mathrm{~km}^{2}$.

\section{Discussion}

Figure 8 suggests that soil moisture is very low in most parts of the region. It ranges from $0.1 \%$ to $25 \%$ and is slightly higher in the northeastern part, while being a bit lower in the southwestern part. Statistical analysis indicates that no more than $10 \%$ of the total region has soil moisture higher than $10 \%$. Scarce precipitation and very high daily evaporation may be the main reasons for the low soil moisture in this region. To the north of two east-west parallel lines is the southern slope of the Altai Mountains, where the terrain slopes gently. The soil here is mainly composed of silty loam, which has a higher water-holding capability and, hence, relatively higher soil moisture compared with the southern part of the line features, where loamy sand distributes widely and the Gobi desert covers a large part. A triangle feature displayed in yellow crosses over the aforementioned line features is also notable. The soil moisture is very low here, which is considered reasonable because this area is occupied by shift sand according to Landsat 8 image interpretation. Meanwhile, the reddish irregular block oriented north and south to the west of the triangle feature is bare rock and, hence, has the lowest soil moisture. In the multishoreline 
areas (as indicated by the red arrow in Fig. 8), soil moisture distributes in a banding appearance, which is in good agreement with the orientation of the multishoreline. Field investigations discovered that these shorelines (more precisely, paleoshorelines) are formed in historical periods and are generally composed of loose sediments; therefore, the water-holding capability is poor and the soil moisture is correspondingly lower. In the western part of Fig. 8, from the multishoreline to the center of the dried-up lake, soil moisture increases first and then decreases because shift sand covered above the salt crust. A few studies to inverse soil moisture based on SAR technology have been carried out in the Ejina alluvial fan and the middle reach of the Heihe river, ${ }^{33,34}$ these studies also corroborate our results. Above all, soil moisture in this region is low, and the inversion result is in reasonable agreement with the factual circumstances.

\section{Conclusions}

Combined with in-situ measured soil moisture, surface roughness, and soil texture, fine RADARSAT-2 data acquired simultaneously with the field investigations in July 2014 were used to simulate the backscattering properties based on AIEM. A combined roughness parameter $R_{s}$ was established and the influences of roughness and soil moisture on the backscattering simulations were discussed. A multiangle empirical soil moisture retrieval model was implemented and validated in Juyanze for the first time. Inversion results show that (1) the empirical model has favorable validity when incidence angles vary from $15 \mathrm{deg}$ to $50 \mathrm{deg}$. The coefficient of determination $R^{2}$ between the inferred and measured soil moisture is 0.775 with an rmse of $0.626 \%$. Taking the surface roughness and the dielectric constant into account simultaneously, the model is proved to have relatively high accuracy and stability. (2) Soil moisture varies from about $0.1 \%$ to $25 \%$ and is no more than $10 \%$ in most parts of this region. The retrieved soil moisture is in reasonable agreement with the factual circumstances. (3) The multiangle empirical soil moisture retrieval model directly relates the Fresnel reflection coefficient and soil moisture and is independent of ground roughness measurements, giving it great potential for future utilization in similar regions.

Currently, it is an important and challenging task to use polarimetric parameters and discriminators for the estimation of surface roughness and soil moisture. Although fully polarimetric RADARSAT- 2 image was acquired in our study and polarimetric parameters were retrieved, the potential use of polarimetric parameters for the estimation of soil moisture was not discussed in this paper as it is the subject of a separate report. In addition, although the validation of the empirical model with AIEM is reasonable in this case, considering the limited number of the RADARSAT-2 data, the model will be further validated using multiangular data in subsequent studies. Finally, it would be advisable to conduct additional research over a wider range of conditions to corroborate these promising results.

\section{Disclosures}

The authors declare no conflicts of interest.

\section{Acknowledgments}

We thank Ming Jin, Guoqiang Li, Fangliang Li, and Haitao Wei from Lanzhou University and Yuan Qi and Zhenting Wang from Cold and Arid Regions Environmental and Engineering Research Institute, Chinese Academy of Sciences, for their support in the field work. Our gratitude also goes to Fei Liu, Yang Li from Chang'an University, Jiawu Zhang from Lanzhou University, and the anonymous reviewers for their constructive suggestions and comments. This work was supported by the National Natural Science Foundation of China under Grant Nos. 41371220, 41571181, and 41272246 and by the Fundamental Research Funds for the Central Universities under Grant No. 0009-2014G2270012.

\section{References}

1. F. T. Ulaby, "Radar measurement of soil moisture content," IEEE Trans. Antennas Propag. 22(2), 257-265 (1974). 
Yang et al.: Implementation of a multiangle soil moisture retrieval model using RADARSAT-2 imagery...

2. J. C. Shi et al., "Estimation of bare surface soil moisture and surface roughness parameter using L-band SAR image data," IEEE Trans. Geosci. Remote Sens. 35(5), 1254-1266 (1997).

3. J. C. Shi and K. S. Chen, "Estimation of bare surface soil moisture with L-band multi-polarization radar measurements," in Int. Geoscience and Remote Sensing Symp. (IGARSS 2005), pp. 2191-2194 (2005).

4. N. Baghdadi et al., "A potential use for the C-band polarimetric SAR parameters to characterize the soil surface over bare agriculture fields," IEEE Trans. Geosci. Remote Sens. 50(10), 3844-3858 (2012).

5. K. C. Kornelsen and P. Coulibaly, "Advances in soil moisture retrieval from synthetic aperture radar and hydrological applications," J. Hydrol. 476(7), 460-489 (2013).

6. G. P. Petropoulos, G. Ireland, and B. Barrett, "Surface soil moisture retrievals from remote sensing: current status, products future trends," Phys. Chem. Earth, Parts A/B/C 83-84, 36-56 (2015).

7. F. T. Ulaby, R. K. Moore, and A. K. Fung, Microwave Remote Sensing Active and PassiveVolume II: Radar Remote Sensing and Surface Scattering and Emission Theory, AddisonWesley, New Jersey (1982).

8. N. E. C. Verhoest et al., "On the soil roughness parameterization problem in soil moisture retrieval of bare surfaces from synthetic aperture radar," Sensors 8(7), 4213-4248 (2008).

9. S. O. Rice, "Reflection of electromagnetic waves from slightly rough surfaces," Commun. Pure Appl. Math. 4(2-3), 351-378 (1951).

10. P. Beckmann and A. Spizzichino, The Scattering of Electromagnetic Waves from Rough Surfaces, Artech House, London, United Kingdom/Norwood, Massachusetts (1987).

11. A. G. Voronovich, Wave Scattering from Rough Surfaces, Springer Science \& Business Media, Germany (1999).

12. A. K. Fung, Z. Li, and K. S. Chen, "Backscattering from a randomly rough dielectric surface," IEEE Trans. Geosci. Remote Sens. 30(2), 356-369 (1992).

13. A. K. Fung and K. S. Chen, "An update on the IEM surface backscattering model," IEEE Geosci. Remote Sens. Lett. 1(2), 75-77 (2004).

14. K. S. Chen et al., "Emission of rough surfaces calculated by the integral equation method with comparison to three-dimensional moment method simulations," IEEE Trans. Geosci. Remote Sens. 41(1), 90-101 (2003).

15. M. S. Moran et al., "Estimating soil moisture at the watershed scale with satellite-based radar and land surface models," Can. J. Remote Sens. 30(5), 805-826 (2004).

16. M. C. Dobson et al., "Microwave dielectric behavior of wet soil-part II: dielectric mixing models," IEEE Trans. Geosci. Remote Sens. GE23(1), 35-46 (1985).

17. J. R. Wang et al., "The SIR-B observations of microwave backscatter dependence on soil moisture, surface roughness, and vegetation covers," IEEE Trans. Geosci. Remote Sens. GE-24(4), 510-516 (1986).

18. Y. Oh, K. Sarabandi, and F. T. Ulaby, "An empirical model and an inversion technique for radar scattering from bare soil surfaces," IEEE Trans. Geosci. Remote Sens. 30(2), 370-381 (1992).

19. P. C. Dubois, J. VanZyl, and T. Engman, "Measuring soil moisture with imaging radars," IEEE Trans. Geosci. Remote Sens. 33(4), 915-926 (1995).

20. Y. Oh and Y. C. Kay, "Condition for precise measurement of soil surface roughness," IEEE Trans. Geosci. Remote Sens. 36(2), 691-695 (1998).

21. N. Baghdadi et al., "Semi-empirical calibration of the IEM backscattering model using radar images and moisture and roughness field measurements," Int. J. Remote Sens. 25(18), 3593-3623 (2004).

22. N. Baghdadi, J. Abou Chaaya, and M. Zribi, "Semi-empirical calibration of the integral equation model for SAR data in C-band and cross polarization using radar images and field measurements," IEEE Geosci. Remote Sens. Lett. 8(1), 14-18 (2011).

23. H. Lievens et al., "Effective roughness modelling as a tool for soil moisture retrieval from C- and L-band SAR," Hydrol. Earth Syst. Sci. 15(1), 151-162 (2011).

24. M. Zribi and M. Dechambre, "A new empirical model to retrieve soil moisture and roughness from radar data," Remote Sens. Environ. 84(1), 42-52 (2003). 
Yang et al.: Implementation of a multiangle soil moisture retrieval model using RADARSAT-2 imagery...

25. F. Yu and Y. S. Zhao, "A new method for soil moisture inversion by synthetic aperture radar," Geomatics Inf. Sci. Wuhan Univ. 35(3), 317-321 (2010).

26. M. Zribi, A. Gorrab, and N. Baghdadi, "A new soil roughness parameter for the modelling of radar backscattering over bare soil," Remote Sens. Environ. 152, 62-73 (2014).

27. J. L. Kong et al., "Retrieval for soil moisture using microwave remote sensing data based on a new combined roughness parameter," Geogr. Geo-Inf. Sci. 32, 34-38 (2016).

28. M. Jin et al., "Holocene shorelines and lake evolution in Juyanze Basin, southern Mongolian Plateau, revealed by luminescence dating," Holocene 25, 1898-1911 (2015).

29. S. Mischke et al., "Mid to Late Holocene palaeoenvironment of Lake Eastern Juyanze (north-western China) based on ostracods and stable isotopes," Geobios 35, 99-110 (2002).

30. T. D. Wu and K. S. Chen, "A reappraisal of the validity of the IEM model for backscattering from rough surfaces," IEEE Trans. Geosci. Remote Sens. 42(4), 743-753 (2004).

31. J. Zeng et al., "A comprehensive analysis of rough soil surface scattering and emission predicted by AIEM with comparison to numerical simulations and experimental measurements," IEEE Trans. Geosci. Remote Sens. 55(3), 1696-1708 (2017).

32. J. M. Wang et al., "Soil moisture content monitoring based on ERS wind scatterometer data," J. China Univ. Min. Technol. 15(4), 305-308 (2005).

33. J. Chen, Y. Jia, and F. Yu, "Soil moisture inversion by radar with dual-polarization," Trans. Chin. Soc. Agric. Eng. 29(10), 109-115 (2013).

34. J. J. Liao and Z. Z. Pang, "Surface parameters retrieval from alluvial fan in Ejina area of inner Mongolia using multi-polarization SAR data," in Int. Geoscience and Remote Sensing Symp. (IGARSS 2009), pp. 498-501 (2009).

Liping Yang is an associate professor at Chang' an University. She received her BS and MS degrees in physical geography from the Northwest University and Lanzhou University in 1992 and 1999, respectively, and her PhD in physical geography from Lanzhou University in 2008. She is the author of more than 30 journal papers. Her current research interests include environmental remote sensing and $3 \mathrm{~S}$ applications.

Yanfei Li received his BS degree in geography from Baoding University and his MS degree in cartography and geographic information system from Chang'an University, respectively. He graduated from Chang'an University, China. His primary research interest is in quantitative estimation of land-surface variables based on active microwave remote sensing.

Qi Li received his BS degree in information management and information system from Shanxi Agricultural University and his MS degree in cartography and geographic information system from Chang'an University, respectively. He graduated from Chang'an University, China. His primary research interest is in environmental remote sensing in arid regions.

Xiaohui Sun received his BS degree in geographic information system from Changchun Normal University and his MS degree in cartography and geographic information system from Chang'an University, respectively. He graduated from Chang'an University, China. His primary research interest is in polarimetric SAR data processing and analysis.

Jinling Kong received her BS and MS degrees in photogrammetry and remote sensing from Wuhan University in 1985 and 1998, respectively and her PhD from Chang'an University in 2004. She is a professor at Chang' an University. Her current research interests include quantitative remote sensing and GIS.

Le Wang received her MS degree in cartography and geographic information system from Chang'an University in 2015. Her primary research interest is in quantitative estimation of land surface temperature. 